PHYSICAL REVIEW A 92, 051401(R) (2015)

$$
\text { S }
$$

\title{
Photon-momentum transfer in multiphoton ionization and in time-resolved holography with photoelectrons
}

\author{
Szczepan Chelkowski, ${ }^{1, *}$ André D. Bandrauk, ${ }^{1}$ and Paul B. Corkum ${ }^{2}$ \\ ${ }^{1}$ Laboratoire de Chimie Théorique, Faculté des Sciences, Université de Sherbrooke, Sherbrooke, Québec J1K 2R1, Canada \\ ${ }^{2}$ Joint Attosecond Science Laboratory of the National Research Council and the University of Ottawa, 100 Sussex Drive, \\ Ottawa, Ontario K1A OR6, Canada
}

(Received 2 July 2015; published 11 November 2015)

\begin{abstract}
In most models and theoretical calculations describing multiphoton ionization by infrared light, the dipole approximation is used. This is equivalent to setting the very small photon momentum to zero. Using numerical solutions of the two-dimensional (2-D) time-dependent Schrödinger equation for one electron (H-like) systems, we show that, for linear polarization, the radiation pressure on photoelectrons is very sensitive to the details of the ionization mechanism. The directly ionized photoelectrons, those that never recollide with the parent ion, are driven in the direction of the laser photon momentum, whereas a fraction of slower photoelectrons are pushed in the opposite direction, leading to the counterintuitive shifts observed in recent experiments [Phys. Rev. Lett. 113, 243001 (2014)]. This complex response is due to the interplay between the Lorentz force and the Coulomb attraction from the ion. On average, however, the photoelectron momentum is in the direction of the photon momentum as in the case of circular polarization. The influence of the photon momentum is shown to be discernible in the holographic patterns of time-resolved atomic and molecular holography with photoelectrons, thus suggesting a new research subject in multiphoton ionization.
\end{abstract}

DOI: 10.1103/PhysRevA.92.051401

PACS number(s): 32.80.Fb, 33.80.Eh, 33.80.Gj, 82.53.Kp

Early multiphoton ionization experiments using intense infrared pulses found the then-amazing result that an ionizing electron often absorbed substantially more photons than the minimum needed for ionization [1]. This puzzling behavior led to the term above-threshold ionization, or ATI, a term still used today. The problem was ultimately solved by computer simulations and the semiclassical recollision model [2-4]. The fate of the associated photon momentum was not even considered until four years ago [5], when it was measured for ultrashort infrared circularly polarized light ionizing neon or argon. That experiment and recent experiments with linear polarization [6] show that the physics associated with the absorbed photon momentum is as equally complex and poorly understood as that with the photon energy. The crux of the problem is that the commonly used (in theory) dipole approximation breaks down in the case of long wavelengths, contrary to the textbook criterion of wavelength $\gg$ atom size $\left(a_{0}\right)$. This criterion is therefore only valid in the perturbative one-photon regime. This breakdown of the dipole approximation is seen at relatively low laser intensities, $I<10^{14} \mathrm{~W} / \mathrm{cm}^{2}$ at which the photoelectrons are still nonrelativistic as shown theoretically in Refs. [7,8]. Having studied the effect of radiation pressure using circular polarization within the strong-field approximation earlier [8], here we solve the time-dependent Schrödinger equation (TDSE) in two dimensions with linear polarization. We report the momentum conservation principle which was missing in the recollision model commonly used in the area.

We find that the average gain of the electron momentum $p_{z}$ (along the beam propagation direction, parallel to $z$-axis) is associated with its kinetic (drift) energy (KE) and only a fraction comes from the atom's ionization energy $I_{p}$.

"S.Chelkowski@USherbrooke.ca
Specifically, we find that

$$
\left\langle p_{z}\right\rangle=\left(\left\langle E_{e l}\right\rangle+0.3 I_{p}\right) / c,
$$

for both linear and circular polarization, where $\left\langle E_{e l}\right\rangle$ is the average photoelectron energy. However, this conclusion is only true for the average, i.e., for the expectation value of the electron momentum $p_{z}$. We show that for linear polarization certain ATI energies gain very different shares of the photon momentum, with some low-energy electrons even reversing sign. These low-energy electrons, showing the reverse (counterintuitive, negative) shifts of the central peak and regular, positive shifts for wings of the spectra, are most easily observed in experiment. In contrast, high-energy electrons $\left(E_{e l}>\right.$ ponderomotive energy) are uniformly shifted in the photon momentum direction. Since circular and linear polarization behave differently, we expect very strong dependence of the results on the ellipticity (and wavelength) of the light.

To solve numerically the TDSE describing the interaction of a hydrogen atom with an intense, linearly polarized laser pulse, we use the velocity-gauge Hamiltonian in which the retardation, $t^{\prime}=t-z / c$, is included in the vector potential $\vec{A}$. We use a two-dimensional (2-D) soft-core model [9] of a $\mathrm{H}$-atom (with regularized 2-D Coulomb potential). In atomic units $\left(m_{e}=\hbar=e=1\right)$ the 2-D laser + system Hamiltonian is

$$
\hat{H}=\frac{1}{2} \hat{p}_{x}^{2}+\frac{1}{2} \hat{p}_{z}^{2}-\frac{1}{\sqrt{r^{2}+0.64}}+A_{x}\left(t^{\prime}\right) \hat{p}_{x}+\frac{1}{2} A_{x}\left(t^{\prime}\right)^{2},
$$

where $r^{2}=x^{2}+z^{2}$ and $\hat{p}_{x}, \hat{p}_{z}$ are the two components of the momentum operator. The laser is linearly polarized along the $x$-axis and propagating along the $z$-axis, and the Gaussian pulse envelope is used, as in Ref. [8]. The total pulse duration is $t_{f}=$ $5 \tau_{\mathrm{FHWM}}$, where $\tau_{\mathrm{FHWM}}=16$ fs is the standard intensity profile width. The same wavelength $\lambda=3.4 \mu$ as in the experiment [6] 


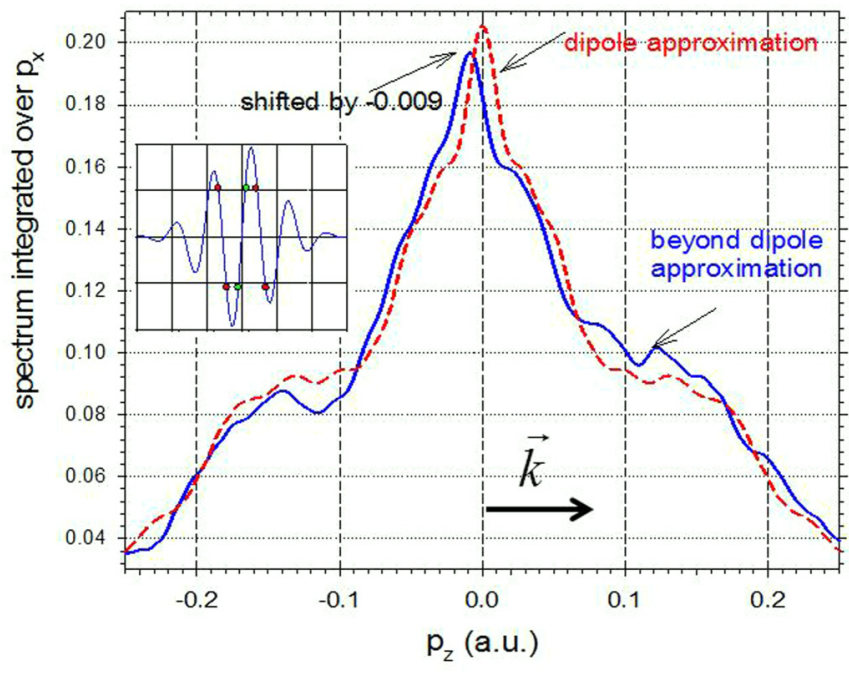

FIG. 1. (Color online) Photoelectron spectra $\tilde{S}\left(p_{z}\right)$ integrated over $p_{x}$. The central cusp is shifted by -0.009 a.u. Laser intensity is $I=10^{14} \mathrm{~W} / \mathrm{cm}^{2}$ and the wavelength $\lambda=3400 \mathrm{~nm}$.

is used. Because of the very long wavelength and long total simulation time, $\sim 96 \mathrm{fs}$, this numerical calculation requires very large grids, which restrict us to the 2-D model. To our knowledge, this is the first TDSE calculation of ATI done for such a long wavelength. The resulting sine-like electric field that we use $E_{x}=-\frac{\partial A_{x}}{\partial t}$ is shown in Fig. 1 (in the inset).

We solve numerically the TDSE using the Fourier split-operator method [10] on a rectangular grid of size $|x|<6144$ a.u. $\gg \alpha=E_{0} / \omega^{2}=353$ a.u., $|z|<1536$ a.u., $\Delta z=\Delta x=0.25$ a.u., i.e., 49152 and 12288 grid points are used along the $x$-axis and $z$-axis directions, respectively. This grid is large enough to minimize the absorption on the grid edge where the absorbing potential is introduced. The system is initialized in the ground $l s$ state and the Schrödinger equation is integrated in time over the duration of the pulse. The time step used in the simulations is $\Delta t=0.03$ a.u. $=0.72$ as. At the end of the pulse, $t=t_{f}$, we calculate the photoelectron momentum ATI spectrum $S\left(p_{x}, p_{z}\right)$ from the spatial Fourier transform of the masked wave function as in Ref. [11].

Figure 1 shows the photoelectron $\tilde{S}\left(p_{z}\right)$ spectra, obtained via the integration of $S\left(p_{x}, p_{z}\right)$ over all $p_{x}$ values where $p_{x}$ is the momentum component along the laser polarization. The red (gray) dashed line is obtained, from TDSE, using the dipole approximation, whereas the solid blue (gray) line shows the results obtained using the exact, beyond-dipole approximation Hamiltonian [Eq. (2)]. Clearly, the resulting exact spectrum shows a surprising twist: The central Coulomb cusp of the exact $p_{z}$ spectrum is antiparallel to photon $\vec{k}$ momentum (negative) shift, that has been experimentally observed [6], whereas the electrons having the momentum component $p_{z} \simeq 0.1$ show the inverse tendency. That is, we observe more electrons with $p_{z} \simeq 0.1$ than with $p_{z} \simeq-0.1$. Overall, this last asymmetry prevails and leads to the positive expectation values $\left\langle p_{z}\right\rangle$ shown in Fig. 2(a), where it is plotted as function of the laser intensity.

Figure 2(a) plots the average $p_{z}$ momentum given by linearly polarized infrared light to the photoelectron in black. It is compared with calculated results [8] for circular polarization

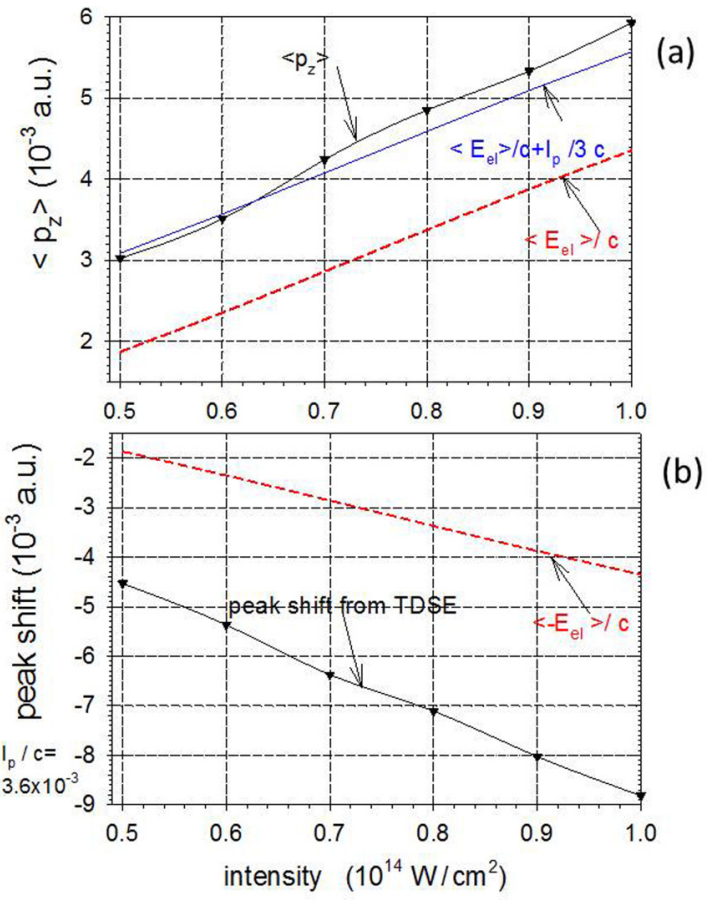

FIG. 2. (Color online) (a) Expectation value of the electron momentum $p_{z}$ and (b) the peak shift, both as function of laser intensity.

(blue [gray]). For reference, we plot the photon momentum associated with $E_{e l}$ with the red (gray) dashed line. Despite the negative shift of the central cusp, the expectation value of $p_{z}$ calculated using the spectrum $\tilde{S}\left(p_{z}\right)$ is positive and its value is similar to that seen for circular polarization [8]. The shift by $I_{p} / 3 c$ is already predicted from the relativistic tunneling theory and is related to the Lorentz force on the bound electron, which lowers the potential barrier [12]. Figure 2(b) shows the negative shifts of the central Coulomb cusp as functions of the laser intensity which are close to experimental results [6].

The contour graphs of the resulting electron momentum spectra $S\left(p_{x}, p_{z}\right)$, using the laser intensity $\mathrm{I}=10^{14} \mathrm{~W} / \mathrm{cm}^{2}$, are shown in Figs. 3 and 4. As seen in Fig. 4 the spectra show structures on the extremely fine momentum scale of $d p_{x} \sim 0.001, d p_{z} \sim 0.01$. Therefore the contour graphs can be plotted only in small portions, as in Fig. 4. To view the spectrum over its entire range we plot low-resolution spectra as contour graphs in Fig. 3. More specifically, Figs. 3(a) and $3(\mathrm{~b})$ show the low-resolution spectra $S_{\text {lowr }}\left(p_{x}, p_{z}\right)$ in which the resolution in the $p_{x}$ variable was decreased to $\Delta p_{x}=0.01$ a.u. Figure 3 compares the low-resolution spectra obtained using the dipole approximation [Fig. 3(a)] with the spectra obtained using the exact Hamiltonian (2) [Fig. 3(b)] and their difference [Fig. 3(c)].

Two prominent features of the figure require discussion. First, we see a strong asymmetry along the electric field direction $\left(p_{x}\right)$. This asymmetry is well understood [13]. It is caused by the carrier envelope phase of the very short pulse that we use. Therefore, we will ignore it and show in Fig. 4 only the positive direction of $p_{x}$.

The second prominent feature shows up when we compare Figs. 3(a) and 3(b). The parallel-antiparallel to $\vec{k}$ symmetry (with respect to the $p_{z}$ momentum component) is broken 


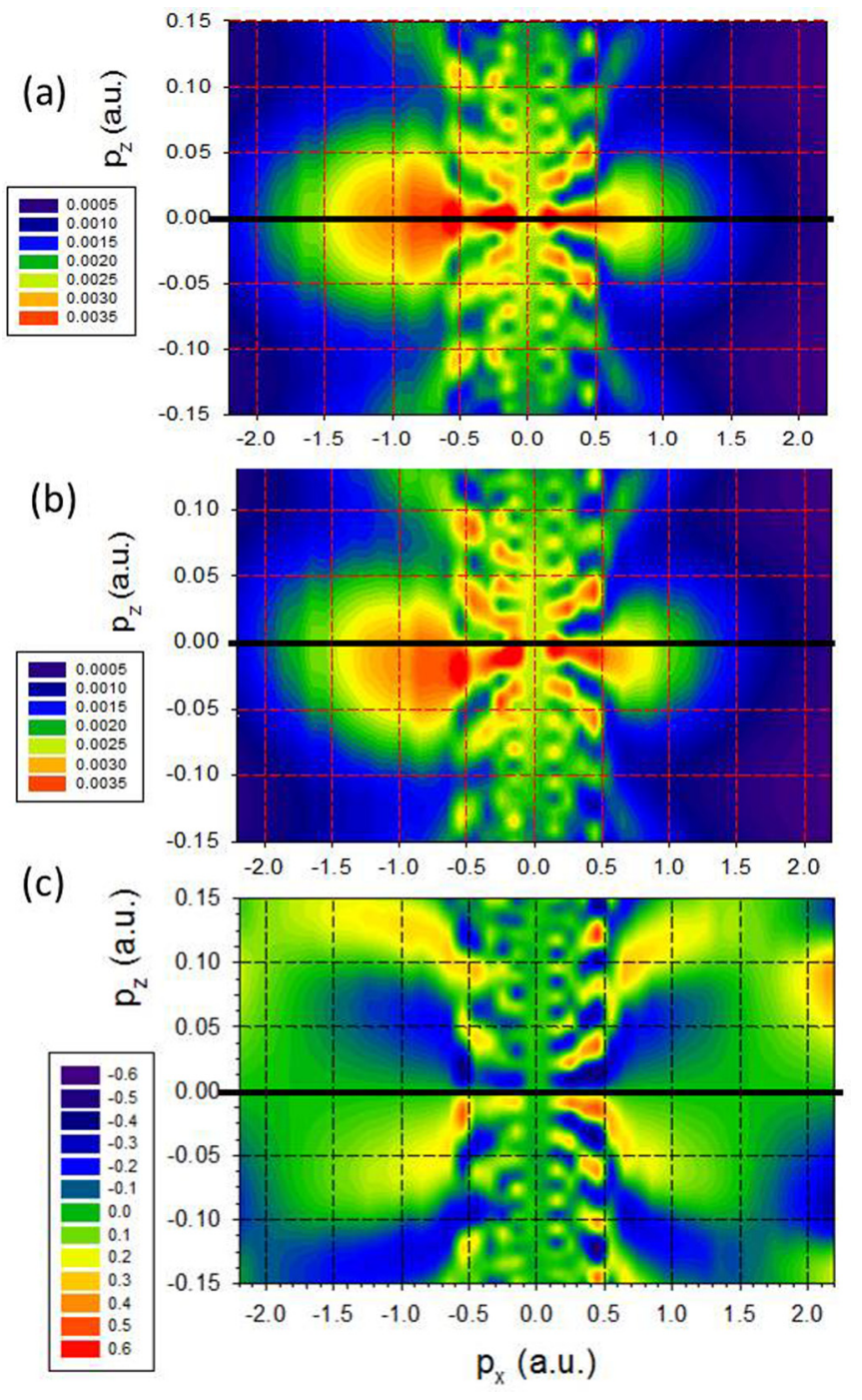

FIG. 3. (Color online) Low-resolution photoelectron spectra, (a) dipole approximation, (b) exact, and (c) asymmetries.

in Fig. 3(b). The strongest (negative) $p_{z}$ shift of the central horizontal crest is seen in the interval $0.6<\left|p_{x}\right|<0.8$. We concentrate on that asymmetry in Fig. 3(c) where the normalized difference $A=\left[S_{\text {lowr }}\left(p_{x}, p_{z}\right)-S_{\text {lowr }}\left(p_{x},-\right.\right.$ $\left.\left.p_{z}\right)\right] /\left[S_{\text {lowr }}\left(p_{x}, p_{z}\right)+S_{\text {lowr }}\left(p_{x},-p_{z}\right)\right]$ is displayed. Very large asymmetries, up to $|A|=0.6$, are seen in Fig. 3(c) while the dipole approximation predicts symmetry, i.e., $A=0$. Note that for small $\left|p_{x}\right|<0.8$ a.u. and for very small $\left|p_{z}\right|<0.02$ the asymmetry shows that more electrons have antiparallel $p_{z}$ momentum, whereas nearly all electrons with larger $\left|p_{z}\right|>0.05$ are parallel to propagation direction momenta. In particular, the strongest asymmetry, opposite to that above, favoring the electrons in the photon $\vec{k}$ direction, is seen for the fast electrons $\left(\left|p_{x}\right| \sim \sqrt{2 U_{p}}\right)$ leading to the shifts $\sim U_{p} / c$, $U_{p}=4$ a.u. for the parameters used in Figs. 3 and 4 . They show clear positive shifts proportional to the electron kinetic energy, $E_{e l} / c$, as expected from classical dynamics [5]. This novel complex symmetry breaking has not been considered in tomography experiments $[14,15]$, where symmetry is often assumed.

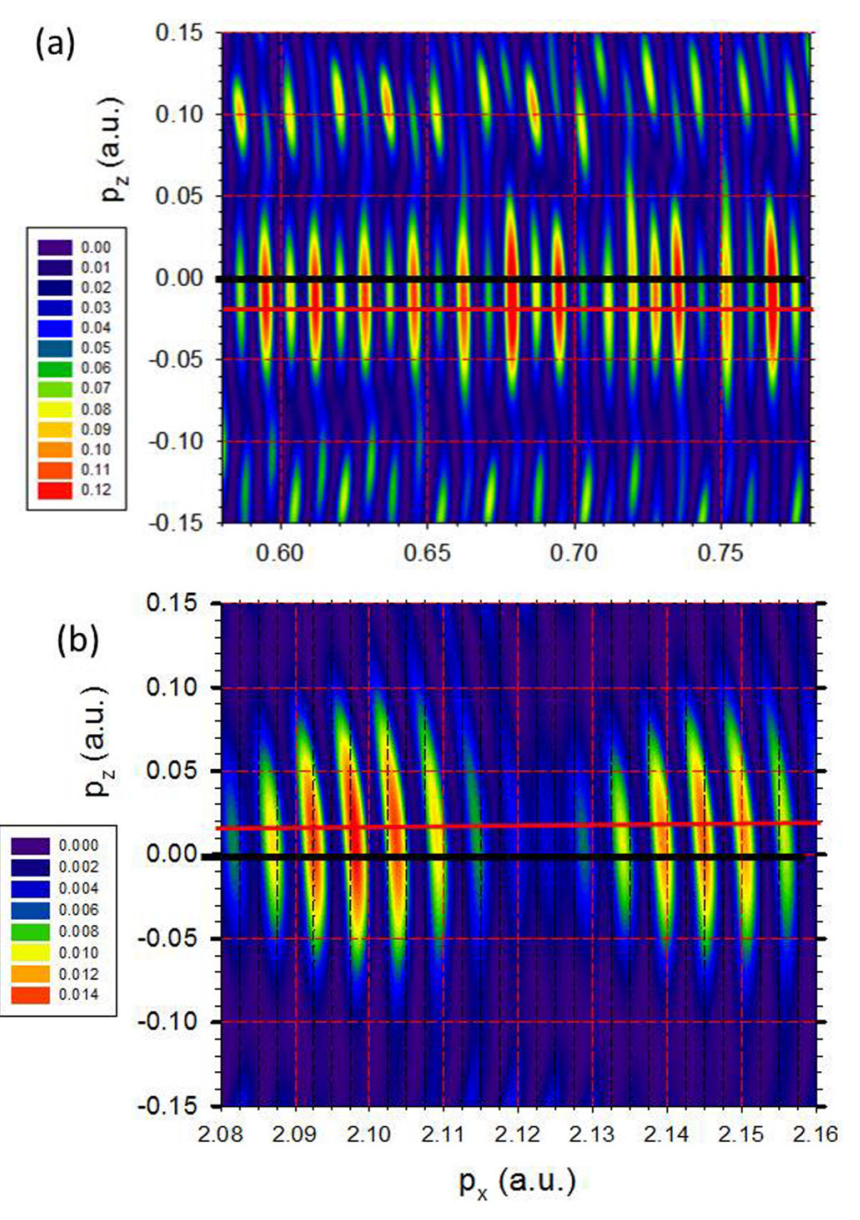

FIG. 4. (Color online) Detailed (high-resolution) contour plots of the photoelectron spectra: (a) slow electrons and (b) fast electrons. Black horizontal lines indicates where $p_{z}=0$ and red (gray) lines show the displacement of central ridges with respect to the dipoleapproximation result.

We plot in Fig. 4(a) the detailed high-resolution spectra corresponding to the momentum interval $0.6<p_{x}<0.8$ showing the strongest antiparallel $p_{z}$ shift. In contrast, Fig. 4(b) shows that the fast electrons, $p_{x}>2$ a.u., are shifted in the classically expected direction for direct electrons and the magnitude of the shift is very close to the classical prediction $p_{x}^{2} / 2 c[5]$.

We also performed classical calculations, similar to those in Refs. $[6,16]$, which allow us to better understand the TDSE results and to identify the trajectories which lead to the opposing shifts. We solve Newton's equation of motion for the electrons which have tunneled at a specific time $t_{0}\left(t_{0}=0\right.$ at the maximum of the electric field). We assume that at $t=t_{0}$ the electron initial position is $z=0, x=x_{0}$ (the coordinate at the exit from the barrier) and the initial momentum component $p_{x}=0$ and nonzero component of the initial $p_{z}$ is given by a Gaussian distribution as in Refs. [6,16]. In Refs. [6,16] Monte Carlo classical spectra $S\left(p_{z}\right)$ were obtained via averaging over all tunneling times $t_{0}$ and all possible initial conditions. In the present work, we select the $p_{x}$ components through their moments of birth [4] $t_{0}$. Similar to Fig. 4, in Fig. 5 we isolate different $p_{x}$ momentum components. In Fig. 5 

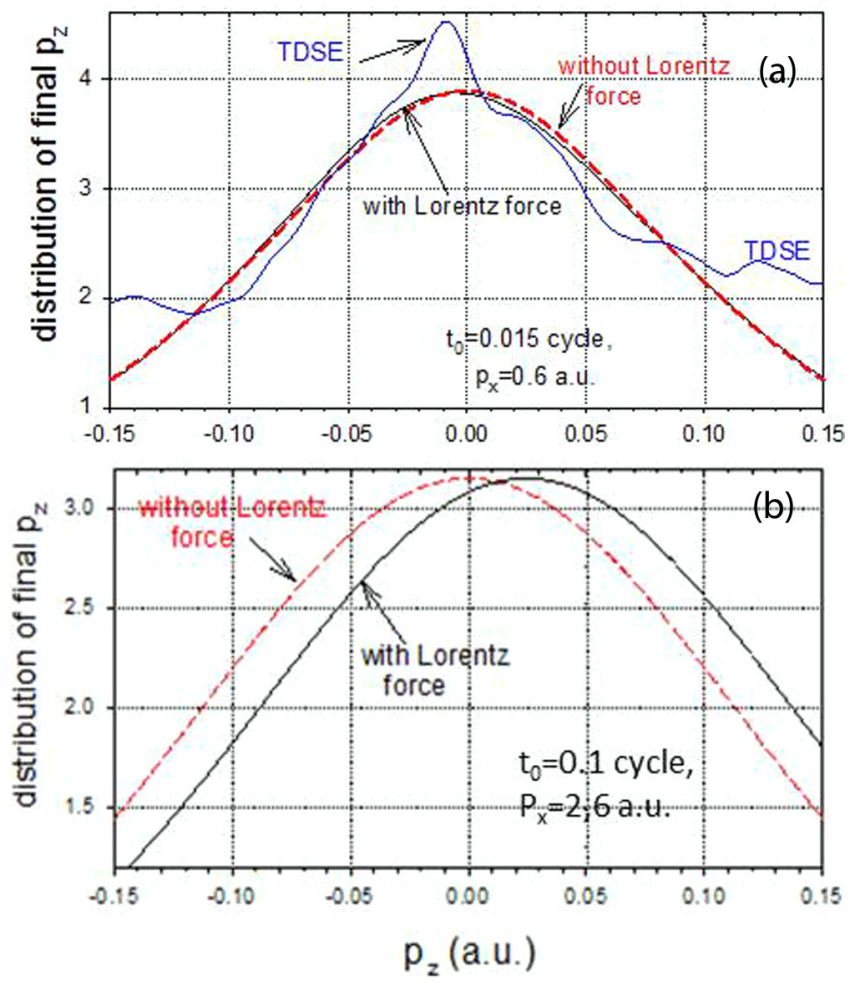

FIG. 5. (Color online) $P\left(p_{z}\right)$ distribution obtained using classical calculations with Lorentz force + Coulomb attraction included (solid lines) and without Lorentz force (dashed lines), for two different tunneling times: (a) $t_{0}=0.015$ and (b) $t_{0}=0.1$ cycle after the electric field maximum, 1 cycle $=11.34$ fs. For comparison, the spectrum $S\left(p_{z}\right)$ from Fig. 1(a) obtained using TDSE is shown in panel (a) (thin blue [gray] line).

we show the classical distributions of final electron momenta $p_{z}$, for the electrons which recollide with the core for two chosen birth times $t_{0}=0.015$ and $0.1 \mathrm{a}$.u. corresponding to the final $p_{x}=0.6$ and 2.5 a.u. values, respectively. The central peak, corresponding to the long trajectory $\left(t_{0}<0.05\right.$ cycle) plotted in Fig. 5(a) shows a negative, antiparallel to $\vec{k}$ shift, similar to our TDSE results shown in Fig. 1, and replotted in Fig. 5(a) (blue [gray] lines). By contrast, for larger $t_{0}$ values, corresponding to faster electrons (and to short trajectories, $t_{0}>0.05$ cycle) Fig. 5(b) shows the positive shifts of the whole $p_{z}$ distributions.

In summary, a simplified intuitive picture emerges for the experimentally and theoretically observed complex momentum distribution along the laser beam propagation direction. Upon ionization, the electron is first pushed in the beam propagation direction by the magnetic field component of light. However, for specific birth times, it can also be deflected into the opposite direction by the Coulomb potential when the electron subsequently passes by the parent ion. We have shown that this antiparallel shift of the central Coulomb cusp occurs mainly for the long-quantum-orbit electrons $\left(t_{0}<0.05\right.$ cycle), i.e., for slow electrons $\left(p_{x}^{2}<U_{p} / 3\right.$ and $p_{z}<0.1$ a.u.). The faster electrons, related to the short quantum orbits, do not show the above effect since it takes for them a shorter time to return to the core. Obviously, the direct electrons (i.e., those components of the electron wave packet that do not recollide) are shifted parallel to the photon momentum as expected from the standard radiation pressure effects.

In the 1930's, Arnold Sommerfeld showed [8,17] that the momentum sharing between the electron and ion in single-photon ionization was, to modern eyes, counterintuitive. The electron gained more than the total photon momentum. This is very different from what we find for multiphoton ionization where the electron gains on average less than the momentum of the absorbed photons. Clearly there is a rich physics to explore between one- and many-photon ionization. In the multiphoton limit, we have shown that, on average, the electron gains the same fraction of the photon momentum for linear and circular. However, for linear polarization, different ATI peaks gain different momenta, some even reversing their average direction. This complex momentum structure breaks the symmetry usually assumed in small-angle holography experiments $[14,15]$. We propose that small-angle holography will be an ideal tool for studying this important effect. These experimental studies will allow us to uncover the rich physics that originates from electron-ion interaction during the ionization process. Classical simulations show how this occurs in the many-photon limit. They will be of less use if only a few photons are involved.

Recently static $[14,15]$ and time-resolved [18] multiphoton holography with photoelectrons has been explored. These forms of atomic or molecular holography rely on the interference patterns and fringes of direct electrons (i.e., those which move directly to the continuum after tunneling) and electrons that tunnel in the opposite direction but return to the core, rescatter through a large angle by the Coulomb potential, and reach the continuum with the same final momentum as direct electrons $[14,15,18,19]$. Our results show that such holographic interference fringes will exhibit clear signatures of the combined effect of Coulomb focusing and the transferred photon momentum to the ionized electron and the parent ion, thus suggesting a future important research direction in molecular imaging [20].

\section{ACKNOWLEDGMENTS}

We thank NSERC for supporting ultrafast science programs and also Compute Canada-Calcul Québec for access to supercomputers.
[1] P. Agostini, F. Fabre, G. Mainfray, G. Petite, and N. K. Rahman, Phys. Rev. Lett. 42, 1127 (1979).

[2] K. J. Schafer, B. Yang, L. F. DiMauro, and K. C. Kulander, Phys. Rev. Lett. 70, 1599 (1993).

[3] P. B. Corkum, Phys. Rev. Lett. 71, 1994 (1993).
[4] P. B. Corkum, N. H. Burnett, and F. Brunel, Phys. Rev. Lett. 62, 1259 (1989).

[5] C. T. L. Smeenk, L. Arissian, B. Zhou, A. Mysyrowicz, D. M. Villeneuve, A. Staudte, and P. B. Corkum, Phys. Rev. Lett. 106, 193002 (2011). 
[6] A. Ludwig, J. Maurer, B. W. Mayer, C. R. Phillips, L. Gallmann, and U. Keller, Phys. Rev. Lett. 113, 243001 (2014).

[7] H. R. Reiss, Phys. Rev. A 87, 033421 (2013).

[8] S. Chelkowski, A. D. Bandrauk, and P. B. Corkum, Phys. Rev. Lett. 113, 263005 (2014).

[9] M. Protopapas, D. G. Lappas, and P. L. Knight, Phys. Rev. Lett. 79, 4550 (1997).

[10] A. D. Bandrauk and H. Shen, J. Chem. Phys. 99, 1185 (1993).

[11] M. Spanner et al., J. Phys. B 45, 194011 (2012).

[12] M. Klaiber, K. Z. Hatsagortsyan, and C. H. Keitel, Phys. Rev. A 73, 053411 (2006); D. Cricchio, E. Fiordilino, and K. Z. Hatsagortsyan, ibid. 92, 023408 (2015).
[13] D. B. Milosevic, G. G. Paulus, D. Bauer, and W. Becker, J. Phys. B 39, R203 (2006).

[14] Y. Huismans et al., Science 331, 61 (2011).

[15] M. Meckel et al., Nat. Phys. 10, 594 (2014).

[16] J. Liu, Q. Z. Xia, J. F. Tao, and L. B. Fu, Phys. Rev. A 87, 041403(R) (2013).

[17] A. Sommerfeld, Atombau und Spectrallinien, Vol. 2 (Braunschweig, Friedrich Vieweg \& Sohn, 1939).

[18] M. Haertelt (private communication).

[19] X. B. Bian and A. D. Bandrauk, Phys. Rev. Lett. 108, 263003 (2012).

[20] L. S. Cederbaum and M. Basler, Phys. Rev. Lett. 103, 133001 (2009). 